\title{
Robótica experimental com uma arquitetura pedagógica para montagem de um sistema de irrigação inteligente
}

\author{
Luiz F. Reinoso ${ }^{1}$, Marcello Amorim², Matheus S. Silva ${ }^{3}$, Eduardo H. Hackbart ${ }^{3}$, \\ Giovany F. Teixeira ${ }^{1}$
}

${ }^{1}$ Coordenadoria de informática - Instituto Federal de Educação, Ciência e Tecnologia do Espírito Santo (IFES)

Colatina - ES - Brasil

${ }^{2}$ Departamento de Informática - Universidade Federal do Espírito Santo (UFES) Vitória - ES - Brasil

${ }^{3}$ Equipe de robótica Asimov -Instituto Federal de Educação, Ciência e Tecnologia do Espírito Santo (IFES)

Colatina, Brasil

luizfreinoso@gmail.com, novaes@inf.ufes.br, matheussoprani@gmail.com, eduardohackbart2000@gmail.com, giovany@ifes.edu.br

\begin{abstract}
This paper presents a pedagogical architecture for the development of intelligent irrigation systems (IIS), which consists of automated mechanisms capable of identifying the absence of rain and the consequent lack of water in the soil. This research aimed to work in an interdisciplinary way, the theme "Economy and human resources management" and the learning of the principles of electronics. This work evaluated the learning curve of the students in disciplines that used, as an additional activity, the development of an IIS based on the proposed architecture.
\end{abstract}

Resumo. Este artigo apresenta uma arquitetura pedagógica para construção de sistemas de irrigação inteligentes (SII), que consistem em mecanismos automatizados capazes de identificar ausência de chuva e consequente falta de água em solo. O objetivo da pesquisa foi trabalhar, de forma interdisciplinar, o tema "Economia e gestão de recursos hídricos" e a aprendizagem de princípios da eletrônica. Foi avaliada a curva de aprendizagem dos alunos em disciplinas que utilizaram, como atividade suplementar, o desenvolvimento de um SII com base na arquitetura proposta.

\section{Introdução}

A robótica educacional vêm sendo utilizada como um recurso pedagógico que permite inserir os estudantes em uma modalidade de ensino diferenciada na qual eles desenvolvem, de forma individual ou colaborativa, soluções que podem ser aplicadas no mundo real. Desta forma eles assumem papéis ativos no processo de aprendizagem, estudando e exercitando suas habilidades de forma prática, o que contribui na evolução em áreas nas quais eles encontram dificuldades.

O município de Colatina é um dos 35 municípios do estado Espírito Santo no Brasil que foram afetados pelos rejeitos da barragem de Mariana no estado de Minas Gerais, o acidente ocorrido em 2015 gerou 1.2 bilhões em prejuízos para o Minas 
VI Congresso Brasileiro de Informática na Educação (CBIE 2017)

Anais do XXVIII Simpósio Brasileiro de Informática na Educação (SBIE 2017)

Gerais com 320.000 pessoas afetadas pela tragédia. No estado de Minas Gerais existem 754 barragens, sendo 317 delas de rejeitos minerários. Dentre diversas medidas tomadas, muitos municípios passaram a fiscalizar, monitorar e restringir o acesso hídrico de indústrias, agricultura e até da população, por segurança, precaução e conscientização (Bottechia, 2017).

O tema “Economia e gestão de recursos hídricos” é uma discussão aberta em diversas disciplinas na instituição em que os estudantes envolvidos neste trabalho estudam. Por este motivo foi o tema escolhido para esta atividade, que teve o objetivo de utilizar a robótica como ferramenta para que os estudantes pudessem desenvolver novos conhecimentos e habilidades ao criar soluções que pudessem ser aplicadas no mundo real. De forma complementar o projeto visou abordar o tema de forma a possibilitar os estudantes construírem uma visão esclarecida de como resolver de maneira inteligente problemas relacionados com desperdício de água e energia na prática da irrigação. Este é um ponto muito importante uma vez que o município passa por uma situação de controle de águas, devido a restrições no uso de recursos hídricos, estabelecidos desde o acidente em Mariana.

David P. Ausubel (2003), no contexto da aprendizagem significativa, diz que para o aprendizado ser efetivo ele deve ser fundamentado em conhecimentos prévios. Ao inserir o estudante em atividades que o permitam exercitar novos conceitos, estes se tornam mais práticos e significativos, e por consequência são internalizados de forma mais eficaz.

Este trabalho pretende oferecer recursos para promover este tipo de atividade, apresentando uma arquitetura pedagógica que permite aos alunos desenvolverem sistemas de irrigação, que fazem uso de inteligência computacional para monitorar alguns eventos climáticos e condições do solo, assim como para tomar decisões relacionadas com seu funcionamento. O SII desenvolvido no experimento realizado consiste em um mecanismo automatizado que pode identificar ausência ou presença de chuva e falta de água em solo. O experimento foi conduzido em diferentes cenários que incluíram vasos de plantas e áreas de controle.

$\mathrm{Na}$ literatura podem ser encontrados diversos trabalhos relacionados a este, como o apresentado por Matricciani et al. (2016) e a partir do qual foi disponibilizado um modelo de sistema de irrigação automática similar com intuito de promover a gestão hídrica e sustentabilidade para moradias urbanas. Seus estudos avaliaram a melhora na qualidade de hortaliças por meio de decisões baseadas em sensores de temperatura e umidade. Cunha e Rocha (2015) também apresentaram um projeto interessante voltado para a agricultura familiar, no qual estudaram o acionamento automático com base na umidade e temperatura do ar. Teixeira et al. (2016) trabalharam no monitoramento de variáveis ambientais, detalhando o funcionamento e o gerenciamento de recursos baseados em mudanças climáticas, os autores destacaram o fato de que os estudantes que participaram do projeto adquiriram certo grau de conhecimento sobre os principais sensores e formas de monitorar o ambiente.

O SII desenvolvido no experimento foi construído com base no Arduíno® (www.aruino.cc), uma plataforma de hardware muito acessível e amplamente utilizado no meio acadêmico, e à qual é possível conectar os sensores, analógicos e digitais necessários ao projeto. Esta plataforma pode ser utilizada de maneira fácil e segura em sala de aula para criar projetos eletrônicos diversos (Arduino, 2013 e Monk, 2014). 
Este projeto adotou como estratégia trabalhar, de forma interdisciplinar, o tema "Economia e gestão de recursos hídricos" e a aprendizagem de princípios da eletrônica como uso e função de componentes eletrônicos, confecção de placas de circuitos, soldagem e manufatura de dispositivos.

Para adequação e melhor adaptabilidade das atividades e trabalho dos conteúdos abordados em cunho educacional, o trabalho foi adaptado a uma arquitetura pedagógica (AP), com este arcabouço metodológico, foi possível estabelecer objetivos e melhorar a gestão dos recursos digitais e tecnológicos envolvidos.

Os resultados obtidos a partir das avaliações e testes aplicados são apresentados por meio de gráficos e tabelas que ilustram a curva de aprendizagem dos alunos, além de apresentar dados relacionados com o tempo dedicado às atividades necessárias ao trabalho.

O restante deste artigo está estruturado da seguinte forma: a seção 2 descreve a arquitetura pedagógica utilizada; a seção 3 descreve detalhadamente os sistemas de irrigação inteligente autônomos; a seção 4 apresenta a análise dos resultados obtidos; e a seção 5 apresenta as considerações finais e a conclusão do trabalho.

\section{APMSII - Uma arquitetura pedagógica para montagem de um sistema de irrigação inteligente}

Uma arquitetura pedagógica (AP) estrutura a estratégia de ensino de forma que as atividades possam ser realizadas de modo mais livre possível, permitindo que o aluno molde seu conhecimento e aprendizagem. As Arquiteturas Pedagógicas são suportes estruturantes à aprendizagem (Carvalho et al. 2005), focados na construção do conhecimento, de ideias construtivistas, de Jean Piaget (1985) e Paulo Freire (1988).

Uma AP pode ainda ser descrita, de modo pragmático, como uma estrutura constituída de: 1. objetivo pedagógico (o que aprender); 2. atividade(s) pedagógica(s) (o que fazer); 3. método pedagógico (como fazer cada atividade) e 4. recursos digitais (com que suporte). De acordo com esta definição, foi criada para este projeto a arquitetura pedagógica APMSII (Arquitetura Pedagógica para montagem de um sistema de irrigação inteligente), com as seguintes características:

1. Objetivo pedagógico: criar uma solução tecnológica inteligente com uso da eletrônica e disciplinas auxiliares para gestão de recursos hídricos.

2. Atividades pedagógicas: desenvolver um sistema eletrônico de irrigação com solução lógica através da criação de software para funcionamento de sensores e atuadores necessários ao sistema.

3. Metodologia: os alunos usarão a placa de prototipagem Arduino Uno, sensor de chuva YL-83 com comparador LM393, com saídas digital e analógica, sensor de umidade de solo HL-69, Relé SRD-05VDC-SL-C, leds, transistores e fios para criar o sistema de irrigação. Com base nos dados dos sensores os estudantes criarão um programa que interprete os dados e tome ações coordenadas para gerir a irrigação de um local. Antes da montagem os alunos farão teste em sistemas de simulação.

4. Recursos digitais: uso do programa Autodesk Circuits ${ }^{\circledR}$ (circuits.io), que possibilita a prototipagem virtual do sistema antes da montagem física. Para 
criação e transferência do código fonte gerado para o circuito físico, utiliza-se o Arduino IDE, software disponibilizado gratuitamente para o uso da Arduino.

A execução da APMSII foi realizada com uma equipe de quatro alunos que trabalharam, de forma colaborativa, para criar toda a estrutura física e lógica do sistema de irrigação. O professor auxiliar se limitou a intervir para garantir a segurança durante o manuseio de equipamentos, assim como a produzir relatórios das tarefas realizadas pelos estudantes.

Os estudantes não possuíam conhecimento prévio sobre os equipamentos e softwares utilizados, nem foram realizadas capacitações. Desta forma eles tiveram a oportunidade de construir seu próprio conhecimento, sendo o professor apenas um mediador deste processo que envolveu a investigação a partir de sistemas de busca, estudo dos livros que acompanham os kits de desenvolvimento Arduíno, até a interação entre colegas de classe nos moldes sócio-interacionista e construtivista descritos (Vigostki, 1991).

Antes de iniciarem a manipulação e construção dos equipamentos reais, os estudantes construíram um protótipo virtual por meio do ambiente interativo e reativo Autodesk Circuits ${ }^{\circledR}$, que permite projetar e construir simulações de projetos eletrônicos. O Circuits é um projeto da Adobe ${ }^{\circledR}$, gratuito para uso educacional, que oferece suporte ao trabalho colaborativo permitindo o compartilhamento de projetos entre os membros de uma equipe (Santos et al., 2016).

\section{SII - Sistema de irrigação inteligente autônomo}

O Sistema de irrigação inteligente (SII) foi desenvolvido com a placa de prototipagem open-source Arduino Uno. Este equipamento possui um microcontrolador baseado no ATmega328, que pode alcançar até $20 \mathrm{MHz}$, suporta temperaturas de $25^{\circ} \mathrm{C}$ a $85^{\circ} \mathrm{C}$, e possui capacidade de até $32 \mathrm{KBytes}$ de memória para armazenamento de código executável pelo dispositivo. Existe uma variação de 1.8 a $5.5 \mathrm{~V}$, quanto maior a frequência do processamento utilizado maior a voltagem (Atmel, 2015).

O SII foi planejado para operar com múltiplos sensores, por este motivo foi adotada a versão Uno do Arduino ${ }^{\circledR}$, que possui 13 portas digitais e 5 analógicas, o suficiente para conectar todos os sensores necessários para o projeto. O esquema de montagem do SII, com base no Arduino ${ }^{\circledR}$ Uno, é ilustrado na Figura 1. 
VI Congresso Brasileiro de Informática na Educação (CBIE 2017)

Anais do XXVIII Simpósio Brasileiro de Informática na Educação (SBIE 2017)

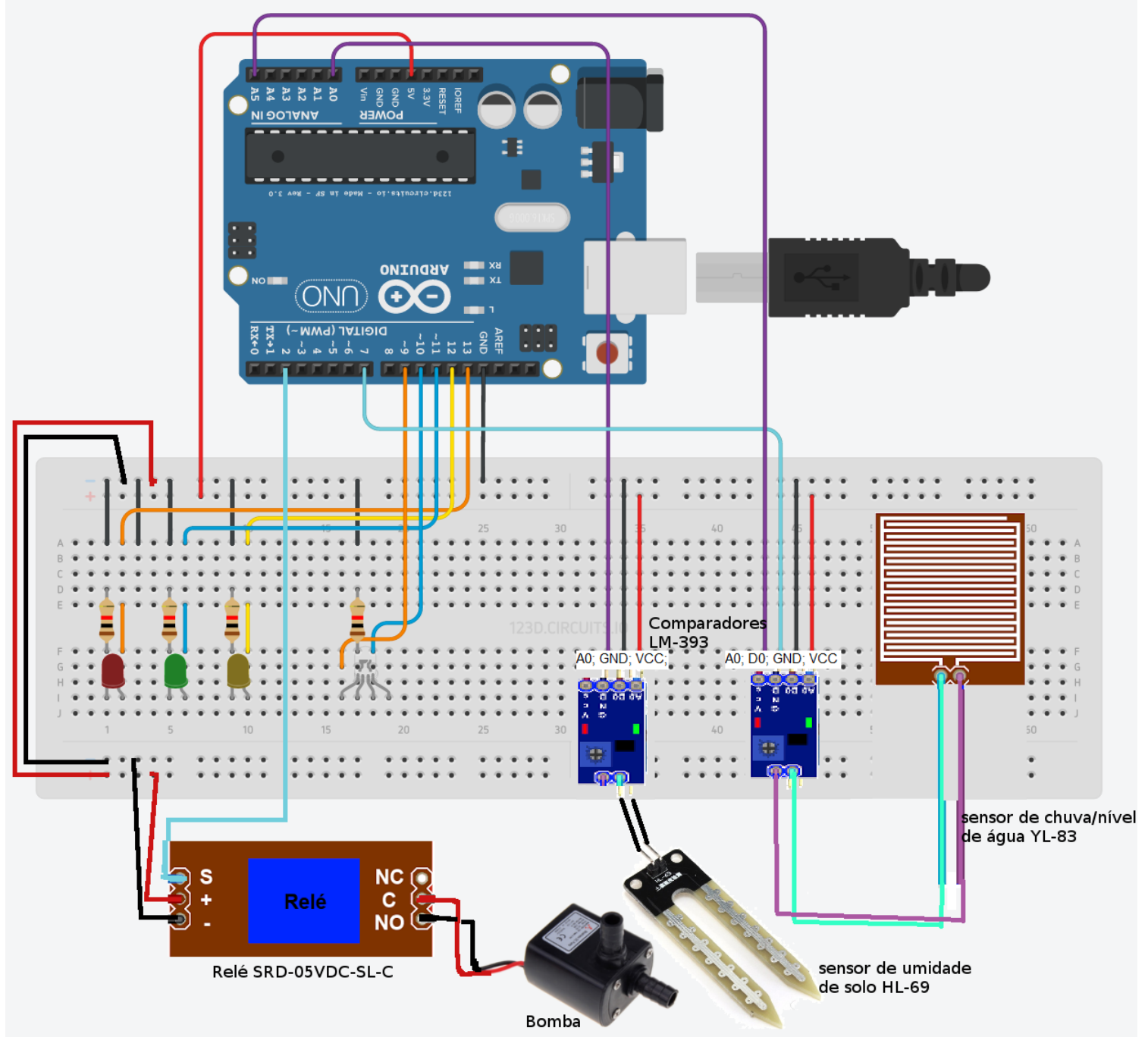

Figura 1. Esquema de montagem do Sistema de irrigação inteligente.

Foram conectados à placa de circuitos integrado: o sensor de chuva YL-83 com comparador LM393, com saídas digital e analógica; o sensor de umidade de solo HL69, Relé SRD-05VDC-SL-C; e os leds indicadores. O led vermelho indica ausência de chuva e umidade em solo, situação na qual era disparado um evento que aciona a bomba de água por meio do relé, com o objetivo de irrigar o solo. O led verde indica a presença de chuva, e o amarelo indica que o nível de água em solo está adequado.

O sensor de chuva funciona como um medidor de resistência, conforme o nível de água no sensor aumenta a resistência diminui e assim ele pode indicar se está chovendo. Caso o nível de chuva indicado pelo sensor seja suficiente para garantir um nível de umidade necessária no solo, o sistema mantém a bomba de água desligada, evitando desperdício de água e energia, assim como desgaste do equipamento.

Para garantir o funcionamento correto, o sensor de umidade foi posicionado no mesmo nível das raízes das plantas, desta forma foi possível identificar o nível de umidade no solo em torno delas. O limiar de ativação inferior do sensor de umidade foi de $80 \%$, valores inferiores a este indicam que as plantas necessitam de mais água no solo. O SII também permite configurar a vazão da bomba de água, assim foi possível 
VI Congresso Brasileiro de Informática na Educação (CBIE 2017)

Anais do XXVIII Simpósio Brasileiro de Informática na Educação (SBIE 2017)

otimizar a quantidade de água utilizada e reduzir o desperdício. No experimento foi utilizada uma vazão de 150 litros/hora. A Figura 2 apresenta o fluxograma do programa que controla o SII:

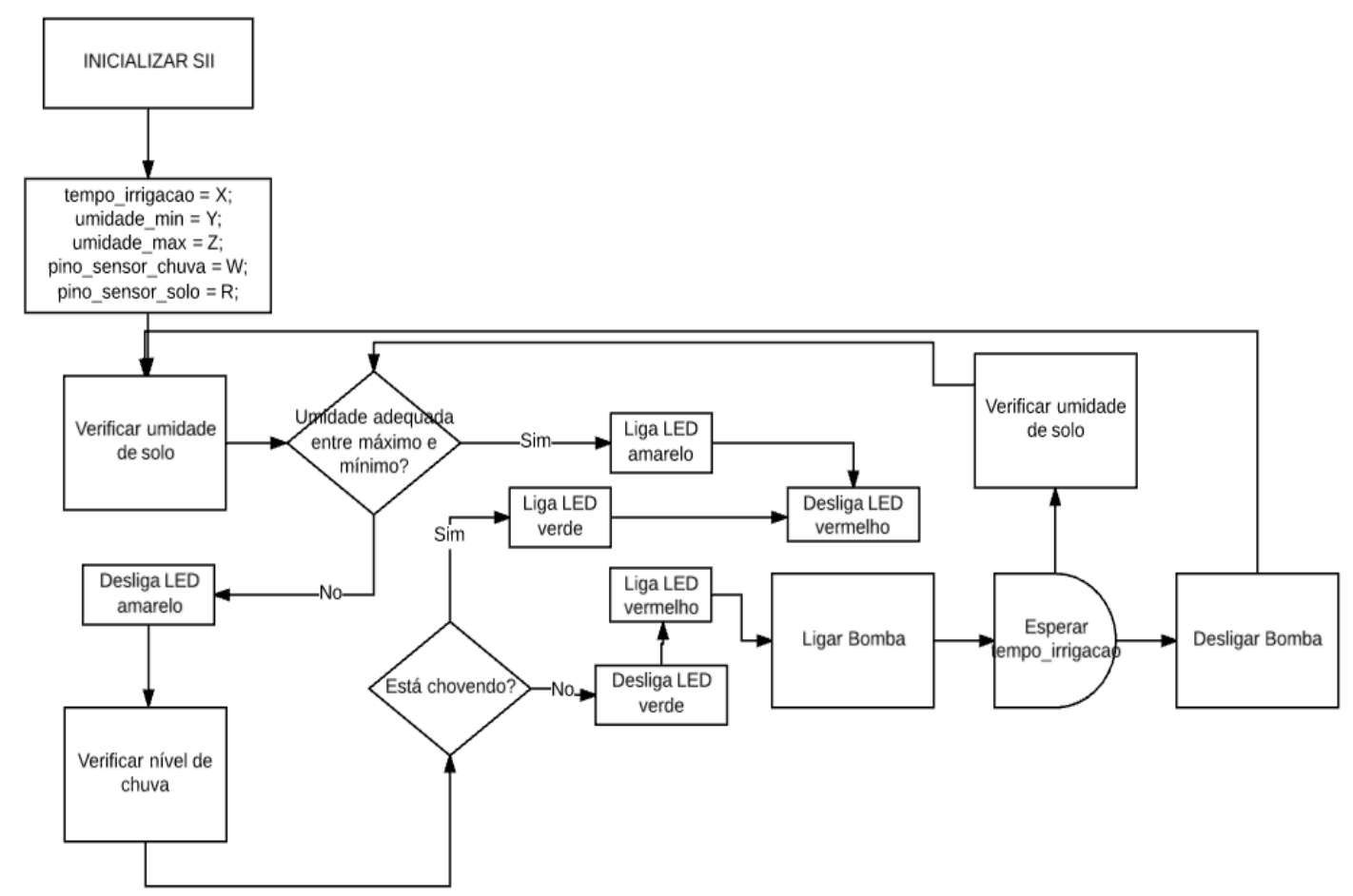

Figura 2. Fluxo de verificação dos sensores e ativação de irrigação.

Para desenvolver a parte lógica do SII os estudantes utilizaram a linguagem de programação $\mathrm{C}++$ e o Arduino IDE, um ambiente integrado de desenvolvimento (Arduino, 2013) fornecido pela empresa fabricante do Arduino®. Esta empresa também disponibiliza bibliotecas e funções padrão que facilitam o desenvolvimento bem como material online em Português e exemplos no site oficial.

De acordo com estas definições, o SII garante que o sistema de irrigação apenas seja ativado caso exista necessidade, que seja utilizada uma vazão adequada de água, e que a bomba de água fique apenas pelo tempo necessário.

Através da programação os estudantes tiveram a oportunidade de testar diferentes valores para os parâmetros referentes ao tempo de irrigação, umidade máxima e mínima. Eles puderam observar que para cada ambiente e cada tipo de planta é necessário reconfigurar o sistema, e que isso pode ser feito por meio do software desenvolvido por eles.

\section{Análise e resultados obtidos}

O SII foi desenvolvido por quatro estudantes em quatro dias. Estes estudantes possuem idades entre 14 e 17 anos, e cursam o ensino médio integrado, dois deles são alunos do curso técnico em administração e os outros do curso técnico em arquitetura. Nenhum deles possuía nenhuma experiência anterior em atividades de programação ou mesmo utilizando softwares que requerem instruções por linha de comando.

O levantamento dos requisitos do SII foi feito utilizando uma metodologia 
VI Congresso Brasileiro de Informática na Educação (CBIE 2017)

Anais do XXVIII Simpósio Brasileiro de Informática na Educação (SBIE 2017)

etnográfica (Cervo et al., 2007), que respeita a inteligência e experiência local. Foram realizadas entrevistas e conversas com professores e alunos. Adicionalmente, os estudantes utilizaram um modelo de ciclo de vida incremental (Pressman, 2011) para desenvolver o SII.

A Figura 3 apresenta, em ordem cronológica, as ações executadas pelos alunos para desenvolver e implantar o SII. Cada ação foi definida e desenvolvida pelos alunos durante a execução da APMSII, e a partir delas os mesmos alcançaram os objetivos explicitados pelo professor, que não intervinha na proposta ou implementação do sistema.

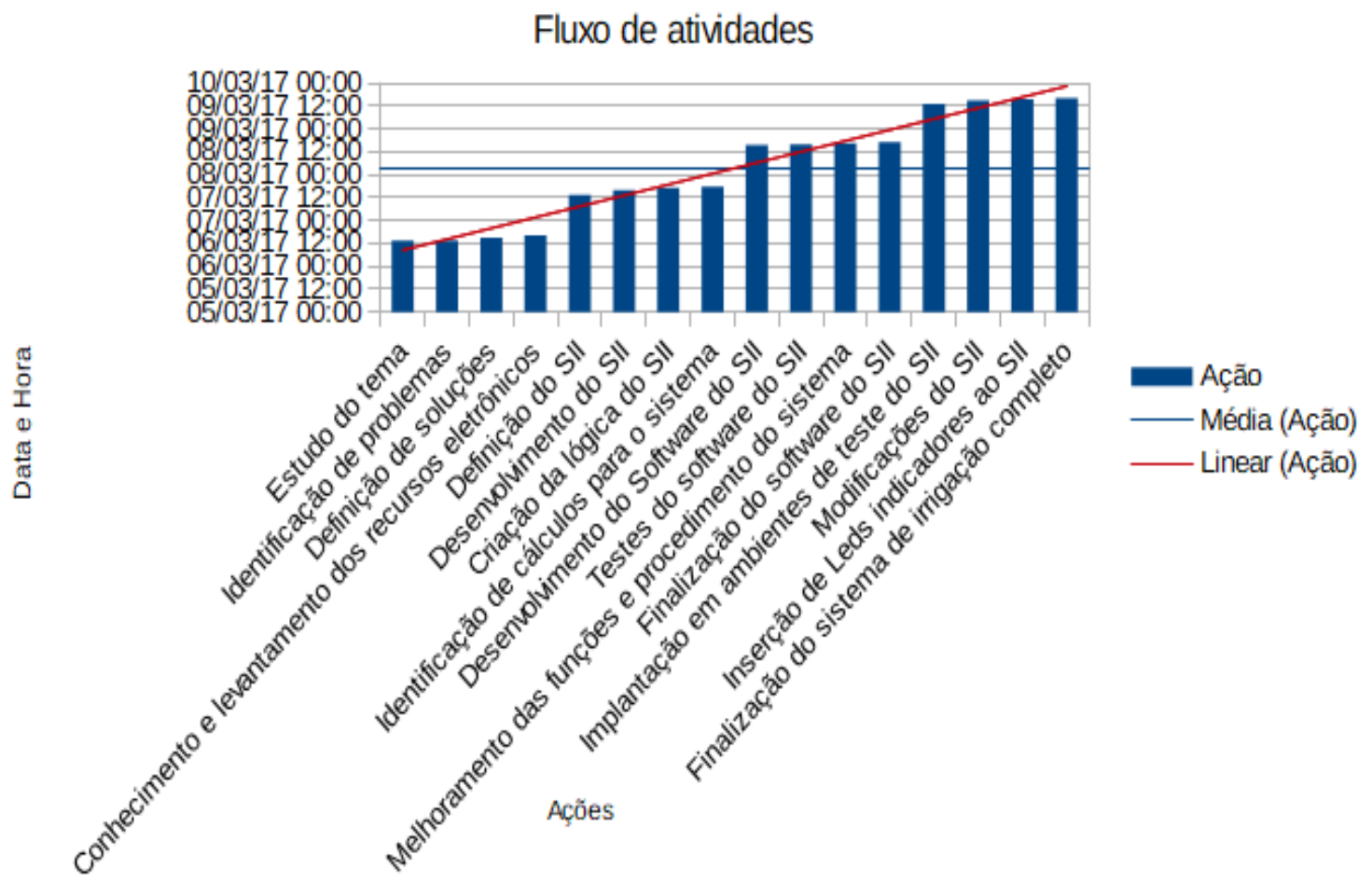

Figura 3. Fluxo de atividades realizadas pelos alunos.

O aprofundamento no estudo do tema ajudou os estudantes a identificarem os recursos eletrônicos que possibilitaram ajudar na construção do SII, em apenas dois dias, um protótipo virtual do SII foi construído por meio do Autodesk Circuits ${ }^{\circledR}$, e em seguida foi reproduzido pelos estudantes como um dispositivo real. A programação estava funcional no terceiro dia, quando foram feitos testes utilizando um vaso de capacidade para 1 litro de água no qual havia uma planta, e também em uma área controlada de solo em ambiente aberto, ambos com o mesmo tipo de substrato.

Durante a execução dos testes os alunos relataram relativa facilidade para instalar e executar o sistema no vaso, pois o ciclo da água bem como ajustes da raiz são bem determinados, tendo no máximo 21 segundos de irrigação (quando o substrato está totalmente seco), devido a pequena área, contabilizando 0,875 litros de água. Todavia, eles perceberam que em um ambiente aberto, a água escorre forma irregular chegando a demorar 58 segundos para a irrigação e consumindo cerca de 2,3 litros de água. Para determinar a configuração adequada foi necessário que estudassem questões como as copas das plantas e a localização da raiz nelas. Após algum esforço eles conseguiram 
VI Congresso Brasileiro de Informática na Educação (CBIE 2017)

Anais do XXVIII Simpósio Brasileiro de Informática na Educação (SBIE 2017)

realizar o experimento em ambiente aberto.

A medida que o código-fonte era criado, os alunos conseguiram identificar regras comuns e aplicáveis nas disciplinas de matemática e física. Os estudantes observaram que a utilização destas regras e propriedades facilitam a implementação e a compreensão do que precisavam e as programavam. A maior evolução neste sentido se dava durante as ações de teste de software e modificações.

Com estes conhecimentos os estudantes conseguiram ajustar o sistema desenvolvido por eles, e a torná-lo mais versátil. Aprenderam a como estruturar e organizar o código-fonte dos programas, possibilitando a reconfiguração da lógica do sistema se necessário a mudança do ambiente a ser monitorado. O software sofreu alterações, gerando três versões até estar completamente funcional.

\section{Conclusão}

O trabalho permitiu o desenvolvimento e aplicação de uma arquitetura pedagógica para a prática da robótica educacional, a APMSII. Todos os objetivos desta AP foram alcançados, e assim foi possível abordar de forma interdisciplinar uma temática importante no contexto regional dos estudantes, assim como contribuir para a sua formação técnica, e gerar um sistema de irrigação inteligente (SII), com infraestrutura de hardware e software gerado por estudantes.

Como resultado, observou-se que à medida que as disciplinas da área de exatas, como matemática e física eram percebidas durante a execução do projeto, os estudantes avançaram rapidamente na programação e montagem do equipamento físico, o que demonstra que a estratégia foi adequada para promover a interdisciplinaridade. O software passou por três versões desde a primeira iteração do projeto, o que mostra o comprometimento dos estudantes com a qualidade dos resultados pretendidos. O sistema completo ficou pronto em apenas quatro dias, e o auge da curva de aprendizagem se deu entre o segundo e terceiro dia, quando foi possível notar a utilização de fórmulas e cálculos matemáticos pelos estudantes.

Para continuidade do projeto e pesquisas futuras, os alunos estudam o uso de placas solares para tornar o SII mais sustentável e autônomo, além do melhoramento contínuo, como recálculo da taxa de irrigação por pluviômetro eletrônico, contrário ao atual uso da umidade como taxa relativa, assim, o sistema passaria a conhecer os milímetros de chuva reais que se encontram em solo.

Todos os artefatos, incluindo o código-fonte, gerados durante este projeto estão disponíveis de forma livre na internet para o público geral, sob o padrão de licença do MIT.

\section{Referencias}

Arduino (2013) “Arduino Projects Book”, 2a . ed. Itália: Torino.

Atmel (2015) “ATmega48A/PA/88A/PA/168A/PA/328/P [DATASHEET]”, pages 1-5. http://www.atmel.com/images/Atmel-8271-8-bit-AVR-Microcontroller-ATmega48A48PA-88A-88PA-168A-168PA-328-328P_datasheet_Complete.pdf

Ausubel, D. P. (2003) “Aquisição e Retenção de Conhecimentos: Uma Perspectiva Cognitiva”, Tradução de Lígia TEOPISTO. $1^{a}$. ed. Lisboa: Paralelo. 
VI Congresso Brasileiro de Informática na Educação (CBIE 2017)

Anais do XXVIII Simpósio Brasileiro de Informática na Educação (SBIE 2017)

Bottechia, J. A. A., Gobira, M. B., Sousa, A. L. M. (2017) "Lições de Mariana e a contaminação do Rio Doce", v.1, n.1. Revista de direito da Administração pública. Niterói: Universidade pages 169-187.

Carvalho, M. J. S., Nevado R. A. and Menezes, C. S. (2005) “Arquiteturas Pedagógicas para Educação a Distância: Concepções e Suporte Telemático. In XVI Simpósio Brasileiro de Informática na Educação”. páginas 351-360, 2005.

Cervo, A. L. Bervian, P. A. and Silva, R. (2007) Metodología Científica, 6a ed. São Paulo: Pearson Prentice Hall.

Cunha, K. C. B. e Rocha, R. V. (2015) “Automação no processo de irrigação na agricultura familiar com plataforma Arduino”, v.01, n. 02. Revista Eletrônica Competências Digitais para agricultura familiar, Tupã, p. 62-74.

Freire, P.(1998) Pedagogia da Autonomia: saberes necessários à prática educativa. Rio de Janeiro: Paz e Terra.

Matricciani, A. R, Nogueira, D. S., G. A. Lima, Marcolino, L. C, Santos, N. B. O, Barboza, R. C., Ishikawa, R. H., Ueno, R. I. B. e Montecin, A. S. A. S. (2016) "Sistema de irrigação automático com Arduino”, v. 22, n. 40, Revista Univap online. http://revista.univap.br/index.php/revistaunivap/article/view/1054/858.

Monk, Simon (2014) “30 projetos com Arduino”, 2a . ed. Porto Alegre: Bookman. 214p.

Piaget, J. (1985). O possível e o necessário - evolução dos possíveis na criança. Porto Alegre, Artes Médicas.

Pressman, R. S. (2011) Engenharia de Software: Uma abordagem Profissional ed. 7, Porto Alegre: AMGH, p. 780.

Santos, O. L., Cury, D., Rafalski, J. e Silveira, P. D. N. (2016) “An IoT Computational Robotics Learning Laboratory in Vila Velha, Espírito Santo”, In: 2016 XI Latin American Conference on Learning Objects and Technology (LACLO). http://ieeexplore.ieee.org/stamp/stamp.jsp?arnumber=7751746.

Teixeira, A. C., Santos, R. P., Rosenbach, L., Nunes, D. G., Silva, R. P., Bugs, C. A. (2016) "Monitoramento de variáveis ambientais por meio de um sistema autônomo baseado na plataforma Arduino”, v.8, n. 2. In: Anais do salão Internacional de Ensino, Pesquisa e Extensão.

Vygotsky, L. S. V. S. (1991) “A formação social da mente”, Tradução de Mônica Stahel M. da Silva. 4". ed. São Paulo: Livraria Martins Fontes Editora Ltda. 